\title{
Schulversagen als \\ ungenügende Kompetenzen \\ im Lesen und Rechnen
}

\section{Philipp Notter}

Der Beitrag betrachtet Schulversagen als ungenügende Kompetenzen im Lesen und Rechnen nach langjährigem Schulbesuch. Er analysiert dazu die Ergebnisse des "International Adult Literacy Survey", der die Lese-und Rechenkompetenzen in der erwachsenen Bevölkerung untersuchte. Zwei Fragen stellen sich: 1. Wie verbreitet sind ungenügende Lese- und Rechenkompetenzen in der Schweiz, und 2. Muss man die Personengruppe, die höchstens eine Ausbildung auf Sekundarstufe I abgeschlossen hat, als Risiko-Gruppe betrachten? Von der einheimischen Bevölkerung weisen ca. 14 Prozent mindestens in einem der drei untersuchten Bereiche für die heutige Gesellschaft ungenügende Kompetenzen auf. Zwar nimmt dieser Prozentsatz bei den jüngeren Personen ab, doch weisen immer noch ca. 6 Prozent der jüngeren Personen mindestens in einem Bereich ungenügende Kompetenzen auf. Bei den Personen, die höchstens eine Ausbildung auf der Sekundarstufe I abgeschlossen haben, weisen ca. 39 Prozent in mindestens einem Bereich ungenügende Kompetenzen auf. Diese Gruppe muss damit als Risiko-Gruppe betrachtet werden.

\section{Einle itung}

Unter Schulversagen versteht man im Allgemeinen verschiedene Schwierigkeiten von Kindern und Jugendlichen in ihrer Schullaufbahn, die zu spezifischen Massnahmen wie Klassenrepetition oder Zuteilung in eine Spezialklasse führen. Man kann Schulversagen jedoch auch in einem etwas weiteren Sinne verstehen. Bei Schulversagen im engeren Sinne geht es ja meistens darum, dass gewisse Lernziele eines Schuljahrs nicht erreicht werden. Man kann nun die Perspektive der einzelnen Schuljahre verlassen und sich die Frage stellen, ob die Lernziele DER SCHULE erreicht wurden. Es ist ein erklärtes und anerkanntes Hauptziel der Volksschule, dass die Kinder Lesen, Schreiben und Rechnen lernen. Schulversagen bedeutet in diesem Sinne, nach langjährigem Besuch der Schule nicht so gut lesen, schreiben und rechnen zu können, wie es erwartet wird. 
Was wird nun erwartet? Was ist nötig, um als Alphabet gelten zu können? Was nötig ist, um als alphabetisiert gelten zu können, unterliegt einem gesellschaftlichen, historischen Wandel (vgl. Hodel, 1985; Giere, 1987). So galt zeitweise jemand, der seinen Namen schreiben konnte, schon als Alphabet. Ein anspruchsvolleres Kriterium ist die Anzahl besuchter Schuljahre. Dieses Kriterium berücksichtigt jedoch die effektiven Lese- und Schreibkompetenzen nicht. Es wurde darum dahingehend präzisiert, dass jemand die durchschnittlichen Leseund Schreibkompetenzen eines bestimmten Schuljahrs, z. B. des dritten Schuljahrs, erreichen musste, um als alphabetisiert zu gelten. Es ist allerdings fragwürdig, ob die durchschnittlichen Lese- und Schreibkompetenzen z. B. eines Drittklässlers reichen, um in den modernen Gesellschaften erfolgreich zu leben. Deshalb wurde in der Diskussion über funktionalen Alphabetismus versucht, die Kompetenzen, die nötig sind, um als funktionaler Alphabet zu gelten, in Beziehung zu den diesbezüglichen minimalen, gesellschaftlichen Anforderungen zu setzen (vgl. Drecoll \& Müller, 1981). Diese minimalen, gesellschaftlichen Anforderungen können allerdings nur sehr unscharf definiert werden.

Neben Schulversagen als ungenügende Kompetenzen im Lesen und Rechnen steht in diesem Artikel ein weiterer Aspekt von Schulversagen zur Diskussion: Aus Nordamerika stammt der Begriff von «drop out», d. h. Jugendlichen, die ohne High-School-Abschluss ihre Ausbildung beenden. Diese Jugendlichen werden in verschiedener Hinsicht als Risiko-Gruppe betrachtet. Auf Schweizer Verhältnisse übertragen entspricht dies Jugendlichen, die ohne einen Abschluss auf der Sekundarstufe II ihre Primär-Ausbildung beenden. Zwar gehört eine Ausbildung auf der Sekundarstufe II nicht mehr zur obligatorischen Schule, jedoch ist sie in der Schweiz die Regel, so dass sich die Frage stellt, ob das Fehlen einer Ausbildung auf der Sekundarstufe II auch als eine Art Schulversagen betrachtet werden kann oder ob diese Jugendlichen aufgrund ihrer Lese- und Rechenkompetenzen zumindest als Risiko-Gruppe zu betrachten sind.

An dieser Stelle muss noch auf ein Problem, das in der Doppeldeutigkeit, die dem deutschen Wort «Schulversagen» inneliegt, zum Ausdruck kommt, hingewiesen werden. Eigentlich könnte das Wort Schulversagen sowohl das «Versagen in der Schule» als auch das «Versagen der Schule» bedeuten. Beim Schulversagen, wie es hier verstanden wird, handelt es sich gewissermassen um ein endgültiges Ergebnis des Schulbesuches. Da stellt sich die Frage, wie weit dieses Ergebnis das Versagen einzelner Individuen oder das Versagen des Schulsystems ist. Im Rahmen dieses Artikels kann diese Problematik nur annäherungsweise angegangen werden, indem untersucht wird, inwieweit «Schulversager» sich durch bestimmte gesellschaftliche (z. B. soziale Herkunft) im Gegensatz zu individuellen Merkmalen auszeichnen. 


\section{Die IALS-Studie}

Im «International Adult Literacy Survey» (IALS) (OECD \& Statistics Canada, 1995; OECD \& HRDC, 1997; OECD \& Statistics Canada, 2000) wurden die Lese- und Rechenkompetenzen der Bevölkerung im Alter von 16 bis 64 Jahren in der jeweiligen Landessprache international vergleichend getestet. Die Studie wurde unter der Leitung von Statistics Canada und der OECD von 1994 bis 1998 in drei Wellen in insgesamt 20 Ländern durchgeführt. Die Schweiz beteiligte sich in der ersten Welle (1994) mit der deutsch- und französischsprachigen Schweiz (Notter, Bonerad \& Stoll, 1999) und in der dritten Welle mit der italienischsprachigen Schweiz. In der deutschen und französischen Schweiz wurde je eine repräsentative Stichprobe von ca. 1400 Personen und in der italienischen Schweiz von ca. 1300 Personen im Alter von 16 bis 64 Jahren untersucht. Die Teilnehmenden wurden zu Hause etwa anderthalb Stunden interviewt. Zuerst wurde ein Fragebogen zum persönlichen Hintergrund, wie Ausbildung, Weiterbildung, Berufstätigkeit und Lesen am Arbeitsplatz und in der Freizeit, ausgefüllt. Danach mussten die Teilnehmenden ca. eine Stunde einen Test in der jeweiligen Landessprache bearbeiten.

Im Test wurden drei Bereiche untersucht: das Lesen von Alltagsprosa, wie Zeitungsartikel, Gebrauchsanweisungen oder Beipackzettel, das Lesen von Alltagsdokumenten, wie Tabellen, Abbildungen oder Formulare, und das Rechnen beim Bearbeiten von Texten, wie z. B. das Addieren von Bestellungen in einem Bestellformular. Die IALS-Untersuchung hat bewusst von einer Zweiteilung der Bevölkerung in Alphabeten und Analphabeten Abstand genommen. Die Lesekompetenzen wurden als ein Kontinuum gesehen, das fliessend vom Nicht-Lesen-Können bis zur Expertise im Lesen reicht. Aufgrund der Ergebnisse im Lesetest wurden drei Skalen gebildet, die von 0 bis 500 Punkten reichen. Ausgehend von einem Modell von Kirsch und Mosenthal (1990), das versucht, die Schwierigkeit des Verständnisses von Dokumenten und Texten empirisch zu erklären, wurden die Skalen in fünf Kompetenzniveaus eingeteilt. Niveau 1 entspricht dabei ungenügender Kompetenz und Niveau 5 sehr guter Kompetenz.

Das unterste Lesekompetenzniveau 1 reicht von Nicht-Lesen-Können bis zu minimalen, ungenügenden Lesekompetenzen. Für die Interpretation der IALSErgebnisse bezüglich des tiefsten Kompetenzniveaus ist es wichtig, eine Vorstellung davon zu haben, wie sehr einfach die Texte und Aufgaben sind, die typisch für dieses Kompetenzniveau sind. So kann z. B. eine Person mit Kompetenzniveau $1 \mathrm{im}$ Lesen von Alltagsprosa aus einem einfachen Beipackzettel eines Medikamentes nicht zuverlässig herauslesen, wie viele Tage man maximal das Medikament einnehmen soll. Eine Person mit Kompetenzniveau 1 im Lesen von Alltagsdokumenten kann z. B. in einem anschaulichen Balkendiagramm nicht zuverlässig einen beschrifteten Balken nach einem bestimmten Kriterium identifizieren. Eine Person schliesslich mit Kompetenzniveau 1 im Rechnen beim Bearbeiten von Texten kann z. B. auf einem Bestellformular nicht zuverlässig 50 
Franken und 2 Franken zusammenzählen. Mit «nicht zuverlässig» ist hier gemeint, dass eine Person auf diesem Kompetenzniveau höchstens eine 80-prozentige Chance hat, Aufgaben auf diesem Schwierigkeitsniveau richtig zu lösen (Beispiele für Texte und Aufgaben finden sich in OECD \& Statistics Canada [1995] und Notter et al. [1999]).

Da die IALS-Studie die Wohnbevölkerung im Alter von 16 bis 64 Jahren untersuchte, unterscheidet sie sich von typischen bildungswissenschaftlichen Studien, die eine bestimmte Schülerpopulation untersuchen. Sie kann darum auch nicht nah an der Schule etwas über Schulversagen aussagen, sondern nur retrospektiv feststellen, was übrig geblieben ist von dem, was man u. a. in der Schule gelernt hat. Des Weiteren beschränkt sie sich von den drei Hauptzielen der Volksschule, lesen, schreiben und rechnen zu lehren, auf das Lesen und Rechnen.

\section{Die Verbreitung von Kompetenzniveau 1 in der Bevölkerung}

Als Indikator für funktionalen Analphabetismus bzw. ungenügende Rechenkompetenzen, also im Sinne dieses Artikels als Indikator für Schulversagen, soll hier das tiefste Kompetenzniveau 1 betrachtet werden. Sogar die besseren auf diesem Kompetenzniveau weisen eine für die Anforderungen in der heutigen Gesellschaft ungenügende Kompetenz auf. Personen auf diesem Lesekompetenzniveau entsprechen im besten Fall dem, was man unter «funktionalen Analphabeten» versteht. In den internationalen Publikationen zur IALS-Studie wird auch noch das Kompetenzniveau 2 als für die Anforderungen der heutigen Gesellschaft ungenügendes Kompetenzniveau betrachtet. Das hier verwendete Kriterium, Kompetenzniveau 1, ist also ein eher zurückhaltendes Kriterium.

In Abbildung 1 ist die Verteilung der im Lande geborenen Bevölkerung von ausgewählten Ländern auf die Kompetenzniveaus in der Prosaskala dargestellt. Da die immigrierte Bevölkerung keine Rückschlüsse auf die Schweizer Schulsysteme zulässt, wird sie in diesem Artikel bei international vergleichenden Analysen ausgeschlossen und bei Analysen, die ausschliesslich auf Schweizer Daten beruhen, nur jener Teil der immigrierten Bevölkerung berücksichtigt, der mindestens die Hälfte der Ausbildung in der Schweiz gemacht hat. Die Ergebnisse der italienischen Schweiz konnten nur bei international vergleichenden Analysen berücksichtigt werden, da noch kein nationaler Bericht vorliegt.

Wie aus Abbildung 1 ersichtlich, gibt es in allen Ländern einen beachtlichen Teil der im Lande geborenen Bevölkerung, der trotz Schulbesuch nicht im eigentlichen Sinn als alphabetisiert betrachtet werden kann. Der Prozentsatz reicht von «tiefen» 5 Prozent in Schweden bis zu 35 Prozent in Italien und 48 Prozent in Portugal. Die Schweiz befindet sich mit ca. 10 Prozent der einheimischen Bevölkerung auf Kompetenzniveau 1 im Mittelfeld. 


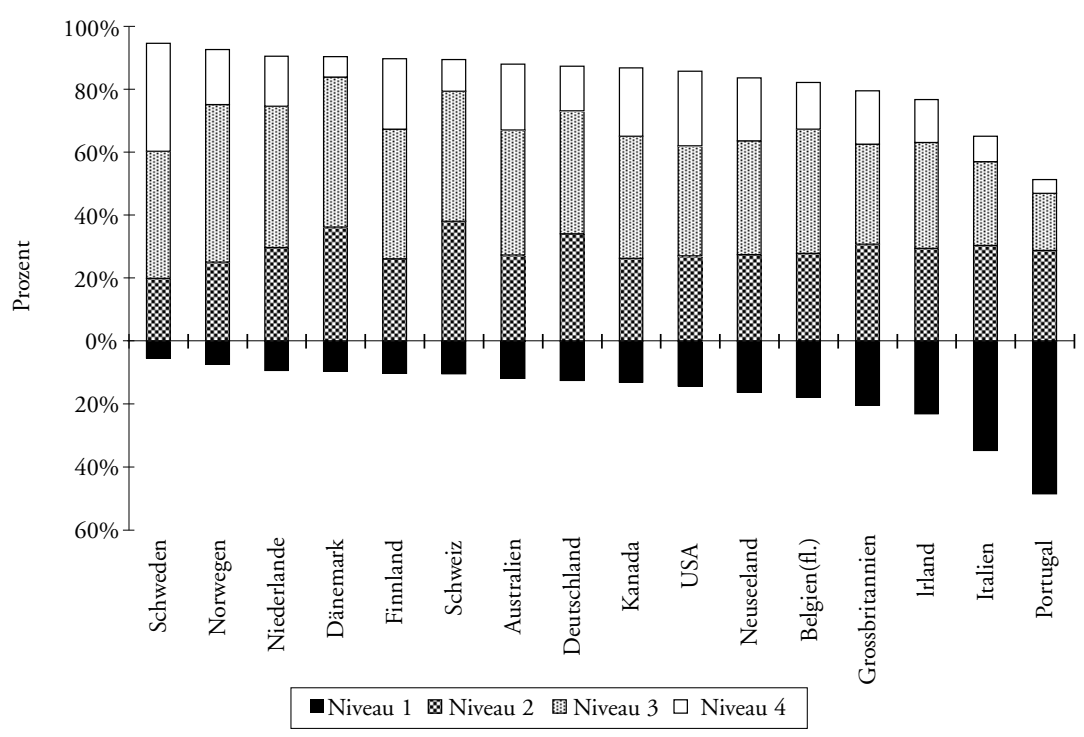

Anmerkung: Schweizer Daten für die deutsche, französische und italienische Schweiz zusammen; die Länder sind nach steigendem Prozentsatz im Niveau 1 geordnet; Quelle: Tuijnman (2001).

Abb. 1: Prozentuale Verteilung der 16-64-jährigen im Lande geborenen Bevölkerung auf die Kompetenzniveaus in der Proskala nach Ländern

Ein sehr ähnliches Bild ergibt sich auch in den Bereichen Lesen von Dokumenten und Rechnen in Texten. Für die Schweiz sind die entsprechenden Prozentsätze der einheimischen Bevölkerung auf dem tiefsten Kompetenzniveau 1 bei der Skala Lesen von Dokumenten ca. 9 Prozent und bei der Skala Rechnen in Texten ca. 6 Prozent. Betrachtet man alle drei Bereiche zusammen, so sind ca. 14 Prozent der einheimischen Bevölkerung in mindestens einem Bereich auf Kompetenzniveau 1 und ca. 4 Prozent sind in allen drei Bereichen auf Kompetenzniveau 1.

Da diese Zahlen die Bevölkerung im Alter von 16 bis 64 Jahren betrifft, haben sie im Hinblick auf das Schulsystem zum Teil in gewisser Weise historischen Charakter. Die ältesten Teilnehmer haben die obligatorische Schule um 1945 beendet und die jüngsten ca. ein Jahr vor der Untersuchung. Es drängt sich darum auf, diese Ergebnisse auch aufgeschlüsselt nach Altersgruppen zu betrachten. Dabei muss man allerdings berücksichtigen, dass in der Zeit seit dem 2. Weltkrieg sowohl die durchschnittliche Ausbildungsdauer als auch das durchschnittliche Ausbildungsniveau gestiegen sind. In Tabelle 1 ist darum die Verteilung auf die Kompetenzniveaus in der Prosaskala nur für die Teilnehmer, deren höchste abgeschlossene Ausbildung auf dem Niveau der Sekundarstufe II ist, nach Altersgruppen aufgeschlüsselt dargestellt. 
Tab. 1: Prozentuale Verteilung auf die Kompetenzniveaus in der Prosaskala nach Altersgruppen für den Teil der Schweizer Bevölkerung, dessen Ausbildung hauptsächlich in der Schweiz stattfand und die mit der Sekundarstufe II abgeschlossen wurde.

\begin{tabular}{|l|c|c|c|c|c|}
\hline Altersgruppen & & & & & Total \\
\hline & Niveau 1 & Niveau 2 & Niveau 3 & Niveau 4/5 & \\
\hline $16-31$ & 3.6 & 37.1 & 47.8 & 11.6 & 100 \\
& $(18)$ & $(186)$ & $(240)$ & $(58)$ & $(502)$ \\
\hline $32-47$ & 7.8 & 40.6 & 43.0 & 8.6 & 100 \\
& $(39)$ & $(203)$ & $(215)$ & $(43)$ & $(500)$ \\
\hline $48-64$ & 16.3 & 45.9 & 32.6 & 5.2 & 100 \\
& $(56)$ & $(158)$ & $(112)$ & $(18)$ & $(344)$ \\
\hline Total & 8.4 & 40.6 & 42.1 & 8.8 & 100 \\
$(113)$ & $(547)$ & $(567)$ & $(119)$ & $(1346)$ & \\
\hline
\end{tabular}

Anmerkung: Daten für die deutsche und französische Schweiz; Personen, die noch in Ausbildung sind, sind ausgeschlossen; in Klammern die Zellenhäufigkeiten.

Die Zahlen in der Tabelle 1 zeigen, dass der Prozentsatz auf Kompetenzniveau 1 von der ältesten Personengruppe zur jüngsten kontinuierlich und massiv von ca. 16 Prozent auf ca. 3 Prozent gesunken ist. Die Gründe für diese Veränderung kann die IALS-Studie nicht klären. In der Diskussion über funktionalen Analphabetismus steht die Hypothese, dass funktionale Analphabeten das Lesen und Schreiben nach der Schulzeit verlernen, der Hypothese, dass funktionale Analphabeten das Lesen und Schreiben nie richtig gelernt haben, gegenüber. Man kann davon ausgehen, dass beide Hypothesen einen Teil des Phänomens erklären. Für die heutige Schule muss man davon ausgehen, dass um die 3 Prozent der Jugendlichen, die ihre Ausbildung auf der Sekundarstufe II abschliessen, im Lesen von Prosatexten nur Kompetenzniveau 1 erreichen. Betrachtet man alle drei gemessenen Bereiche, so erreichen ca. 6 Prozent dieser Jugendlichen in mindestens einem Bereich nur Kompetenzniveau 1. Ca. 5 Prozent erreichen im Lesen von Alltagsprosa oder von Alltagsdokumenten nur Kompetenzniveau 1 und müssen folglich als funktionale Analphabeten, d. h. im Sinne dieses Artikels als Schulversager, betrachtet werden. Diese Zahl entspricht in etwa auch den Schätzungen aufgrund einer anderen Studie, in der im Jahre 1991 die Lesekompetenzen des achten Schuljahres in der Schweiz untersucht wurden (Notter, 1996). 


\section{Die Verbreitung von Kompetenzniveau 1 be $i$ Personen, die nur die obligatorische Schule besucht haben}

In den letzten Jahrzehnten gibt es den Trend, dass die Erstausbildung immer mehr mit einer Ausbildung auf der Sekundarstufe II oder höher abgeschlossen wird, so dass dies heute die Regel ist. In der deutsch- und französischsprachigen IALS-Stichprobe haben z. B. in der Altersgruppe der 56- bis 64-Jährigen noch ca. 27 Prozent ihre Ausbildung auf dem Niveau Sekundarstufe I oder tiefer abgeschlossen, während es in der Altersgruppe der 16- bis 25-Jährigen nur noch ca. 8 Prozent sind. Dabei sind hier alle Personen, die angaben, noch in Ausbildung zu sein, ausgeschlossen.

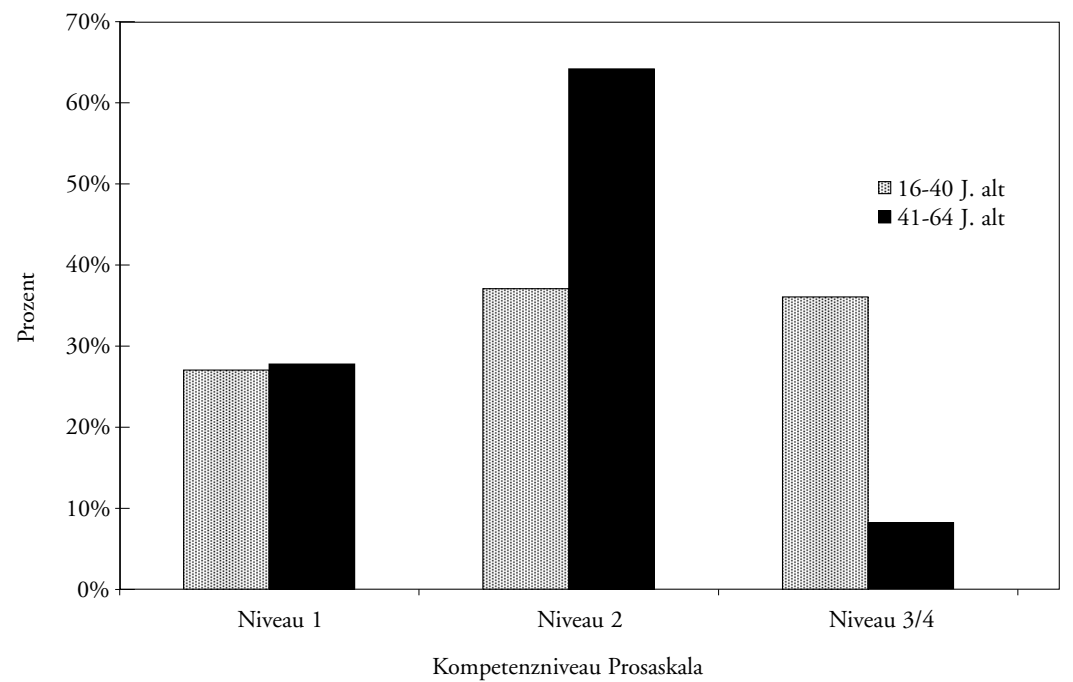

Anmerkung: Daten für die deutsche und französische Schweiz; Personen, die noch in Ausbildung sind, sind ausgeschlossen; $\mathrm{n}$ der Altersgruppe, 16-40 Jahre alt: 100; in der Altersgruppe, 41-64 Jahre alt: 195.

Abb. 2: Prozentuale Verteilung auf die Kompetenzniveaus in der Prosaskala nach Altersgruppen für den Teil der Schweizer Bevölkerung, dessen höchste, abgeschlossene Ausbildung die obligatorische Schule ist.

Wie viel Prozent der Personen, die nur die obligatorische Schule besucht haben, sind nun auf dem tiefsten Kompetenzniveau 1? Im Lesen von Prosa sind 27 Prozent dieser Gruppe auf Kompetenzniveau 1. Im Lesen von Dokumenten sind es sogar 29 Prozent und im Rechnen in Texten sind es 18 Prozent. Betrachtet man 
alle drei untersuchten Bereiche zusammen, so sind ca. 39 Prozent in mindestens einem Bereich auf Kompetenzniveau 1 und ca. 14 Prozent in allen drei Bereichen auf Kompetenzniveau 1. Man kann also zu Recht bei dieser Gruppe von einer Risiko-Gruppe sprechen, haben sie doch eine ca. dreimal grössere Wahrscheinlichkeit, ungenügende Kompetenzen aufzuweisen als der Durchschnitt der betrachteten Bevölkerung.

Doch auch hier muss man, wenn man sich für das heutige Schulsystem interessiert, die historische Perspektive berücksichtigen. Doch ist bei dieser Untergruppe die Stichprobe so klein, dass man die Ergebnisse nicht sehr differenziert nach Altersgruppen aufgliedern kann, sondern sich mit der Unterscheidung zwischen älteren und jüngeren Personen begnügen muss. In Abbildung 2 ist die Verteilung dieser Gruppe auf die Kompetenzniveaus in der Prosaskala nach älteren und jüngeren Personen dargestellt. Dabei wurden Kompetenzniveau 3 und 4 zusammengefasst, weil nur einzelne Personen dieser Gruppe Kompetenzniveau 4 erreichen. Das in Abbildung 2 dargestellte Ergebnis ist erstaunlich. In allen möglichen Vergleichen kann man sowohl national als auch international einen ziemlich kontinuierlichen Alterseffekt feststellen, im Sinne je jünger die Personengruppen, umso höher sind die Lesekompetenzen, wie wir es auch in Tabelle 1 gesehen haben. In Abbildung 2 zeigt sich zwar auch ein Alterseffekt, indem bei den jüngeren Personen prozentual viel mehr auf Kompetenzniveau 3 sind als bei den älteren Personen, aber der Alterseffekt ist hier nicht kontinuierlich, weil der Prozentsatz auf Kompetenzniveau 1 in beiden Altersgruppen gleich ist. Dies scheint nicht ein Effekt der relativ groben Alterseinteilung zu sein, denn auch bei einer differenzierteren und darum wegen der kleinen Anzahl unzuverlässigeren Altersgruppierung bleibt das Erscheinungsbild in etwa dasselbe. Handelt es sich hier in Analogie zur Sockel-Arbeitslosigkeit um einen Sockel-Analphabetismus?

\section{Soziodemographische Merkmale der Personengruppe auf Kompetenzniveau 1}

Auch wenn der Prozentsatz von Personen auf dem tiefsten Kompetenzniveau 1 bei der Gruppe, die höchstens eine Ausbildung auf Sekundarstufe I abgeschlossen hat, am höchsten ist, darf man nicht übersehen, dass Probleme mit Lesen und Rechnen sich nicht auf diese Gruppe beschränken, sondern auf allen Ausbildungsniveaus vorkommen. In Abbildung 3 ist darum links der Prozentsatz auf Kompetenzniveau 1 in der Prosaskala pro Ausbildungsniveau und rechts die prozentuale Verteilung der Ausbildungsniveaus in der Gruppe mit Kompetenzniveau 1 in der Prosaskala dargestellt. Wie aus dem linken Balkendiagramm der Abbildung 3 ersichtlich ist, sind, wie oben erwähnt, ca. 27 Prozent mit abgeschlossener Sekundarstufe I, ca. 8 Prozent mit abgeschlossener Sekundarstufe II und ca. 4 Prozent mit tertiärer Ausbildung auf Kompetenzniveau 1. Da aber die Verteilung auf die Ausbildungsniveaus sehr ungleich ist und rund zwei Drittel 
der Stichprobe ihre Ausbildung auf Sekundarstufe II abgeschlossen haben, hat tatsächlich die Mehrheit der Personen, die im Lesen von Alltagsprosa nur Kompetenzniveau 1 erreichen, eine Ausbildung auf der Sekundarstufe II abgeschlossen. Wie aus dem rechten Balkendiagramm der Abbildung 3 ersichtlich ist, haben von den Personen auf Kompetenzniveau 1 in der Prosaskala ca. 54 Prozent eine Ausbildung auf Sekundarstufe II, ca. 39 Prozent auf Sekundarstufe I und ca. 7 Prozent auf tertiärem Niveau abgeschlossen.

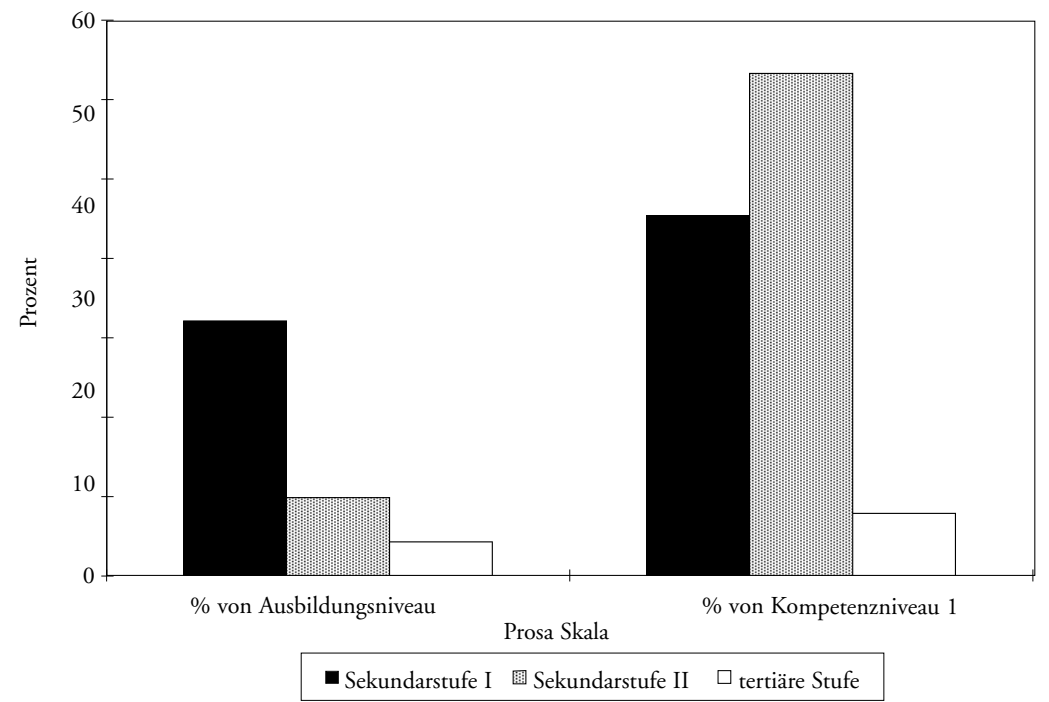

Anmerkung: Daten für die deutsche und französische Schweiz; Personen, die noch in Ausbildung sind, sind ausgeschlossen; n Sekundarstufe I: 295; n Sekundarstufe II: 1348; n tertiäre Stufe: 388; n Kompetenzniveau 1: 208.

Abb. 3: Prozentualer Anteil der Personen auf Kompetenzniveau 1 in der Prosaskala auf der Basis des Totals des jeweiligen Ausbildungsniveaus und auf der Basis des Totals aller Personen auf Kompetenzniveau 1.

Ausser im Alter und in der Ausbildung unterscheiden sich die Personen auf Kompetenzniveau 1 noch im Geschlecht und in der sozialen Herkunft von der übrigen Stichprobe. So sind ca. 62 Prozent der Personen, die in mindestens einem Bereich auf Kompetenzniveau 1 sind, Frauen. Dieses Ergebnis überrascht auf den ersten Blick, zeigte das Geschlecht doch nur einen geringen Einfluss auf die Lese- und Rechenkompetenzen, wenn verschiedene relevante Variablen, wie Ausbildung und Alter kontrolliert werden (s. Notter et al., 1999, S. 59f). Berücksichtigt man jedoch das Alter so zeigt sich, dass diese Übervertretung der Frauen auf die Gruppe der 48- bis 64-Jährigen zurück zu führen ist, bei der der Frauenanteil sogar 75 Prozent ist, während bei den jüngeren Altersgruppen die Geschlechtsverteilung ungefähr ausgeglichen ist. 


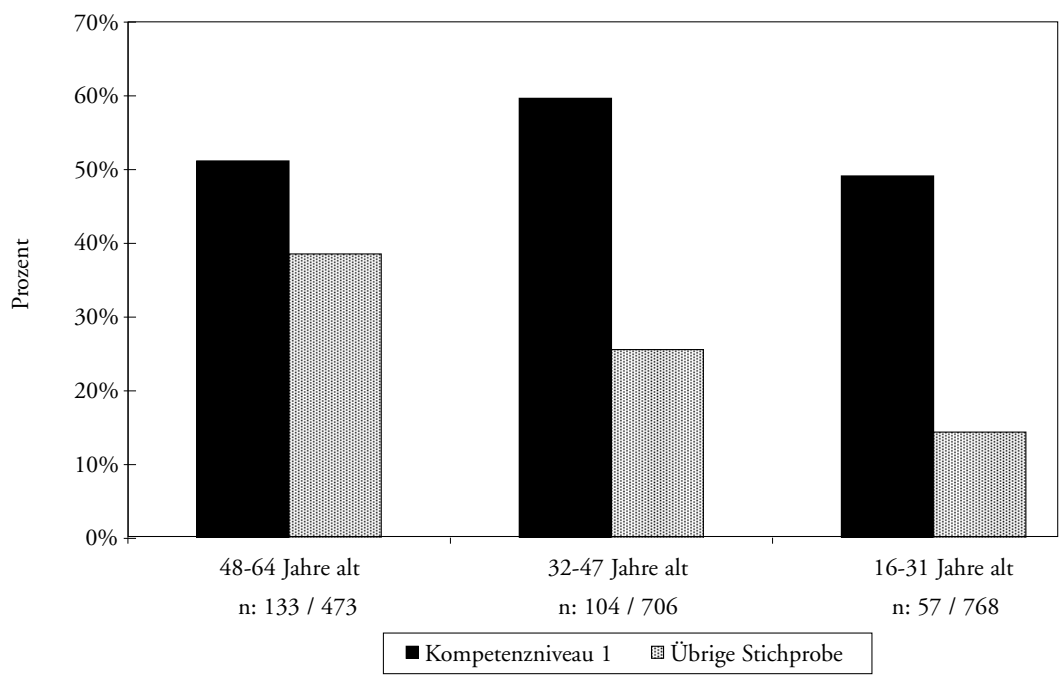

Anmerkung: Daten für die deutsche und französische Schweiz.

Abb. 4: Prozentualer Anteil der Personen, deren Eltern höchstens eine Ausbildung auf Sekundarstufe I haben, für Personen, die mindestens in einem Bereich auf Kompetenzniveau 1 sind, und für die übrige Stichprobe nach Altersgruppen.

In der IALS-Untersuchung wurde das höchste Ausbildungsniveau des besser ausgebildeten Elternteils als Indikator für die soziale Herkunft verwendet. Bei den Personen, die in mindestens einem Bereich nur Kompetenzniveau 1 erreichten, haben ca. 54 Prozent Eltern, die höchstens eine Ausbildung auf Sekundarstufe I abgeschlossen haben, während es in der übrigen Stichprobe nur ca. 24 Prozent sind. Beachtet man auch hier die zeitliche Perspektive durch Berücksichtigung des Alters der Personen, so zeigt sich das in Abbildung 4 dargestellte Bild. Während in der übrigen Stichprobe der Prozentsatz der Personen, deren Eltern höchstens eine Ausbildung auf Sekundarstufe I haben, kontinuierlich von ca. 39 Prozent bei den 48- bis 64-Jährigen auf ca. 14 Prozent bei den 16- bis 31-Jährigen sinkt, bleibt dieser Prozentsatz bei den Personen, die mindestens in einem Bereich nur Kompetenzniveau 1 erreichen, relativ konstant um die 50 Prozent. Einerseits zeigt dies, dass die Bedeutung des Ausbildungsniveau der Eltern als Indikator für die soziale Herkunft sich mit der Zeit gewandelt hat, indem das Merkmal, Eltern zu haben, die höchstens eine Ausbildung auf Sekundarstufe I abgeschlossen haben, bei den 16- bis 31-Jährigen ein viel kleinere, spezifischere Unterschicht kennzeichnet als bei den 48- bis 64-Jährigen. Andererseits bedeutet es parallel dazu, dass das Problem ungenügender Kompetenzen im Lesen und Rechnen schichtspezifischer geworden ist. 
Schliesslich muss noch ausdrücklich auf zwei soziodemographische Merkmale hingewiesen werden, in denen sich die Personengruppe, die mindestens in einem Bereich nur Kompetenzniveau 1 erreicht, nicht von der übrigen Stichprobe unterscheidet: Sowohl in der Nationalität als auch in der Mutter- oder Hauptsprache gibt es keine bedeutsamen Unterschiede zwischen dieser Gruppe und der übrigen Stichprobe. Dies erklärt sich allerdings zum Teil durch die Begrenzung der untersuchten Stichprobe auf jenen Teil der Wohnbevölkerung, der mindestens die Hälfte der Ausbildung in der Schweiz gemacht hat.

\section{Diskussion}

Die Ergebnisse dieser Analyse sind ernüchternd. Wie wir oben gesehen haben, sind ca. 14 Prozent der 16- bis 64-jährigen Wohnbevölkerung, die ihre Ausbildung hauptsächlich in der Schweiz gemacht haben, nach dem hier verwendeten Kriterium Schulversager, weil sie in mindestens einem der untersuchten Bereiche nur Kompetenzniveau 1 erreichen. Da, wie wir oben gesehen haben, die Mehrheit dieser Personen eine Ausbildung auf Sekundarstufe II oder sogar auf der tertiären Stufe abgeschlossen hat, muss man davon ausgehen, dass die durch das hier verwendete Kriterium für Schulversagen identifizierte Personengruppe sich mit Gruppen, die durch andere mögliche Kriterien für Schulversagen gebildet werden, nicht deckt. Bei einem Prozentsatz von 14 Prozent kann man andererseits kaum von einzelnen, individuellen Schulversagern reden, da drängt es sich auf, von einem Versagen des Systems zu reden. Zwar zeigt die Betrachtung der jüngeren Altersgruppen, dass der Erfolg der Schule besser geworden ist, andererseits entkräftet die Tatsache, dass auch bei den Jüngeren ca. 6 Prozent in mindestens einem Bereich nur Kompetenzniveau 1 erreichen, die Hypothese, dass diese Personen Lesen und Rechnen zwar in der Schule gelernt haben, aber nach ihrer Schulzeit wieder verlernt haben.

Die Konsequenzen des Schulversagens, wie es hier definiert wurde, sind für das Individuum und die Gesellschaft, wie anderswo aufgezeigt wurde (Notter et al., 1999), schwerwiegend. So haben Personen auf Kompetenzniveau 1 eine viel höhere Wahrscheinlichkeit, arbeitslos zu werden, sie verdienen bedeutend weniger, sie erleben vermehrt Einschränkungen in ihrer Arbeitskarriere und sie beteiligen sich weniger an Weiterbildung, wodurch sie auch weniger die Möglichkeit haben, ihr Defizit zu überwinden.

Die Analyse der Personengruppe, die höchstens eine Ausbildung auf der Sekundarstufe I abgeschlossen hat, zeigt, dass es sich hier wirklich um eine RisikoGruppe handelt, da ca. 39 Prozent mindestens in einem untersuchten Bereich nur Kompetenzniveau 1 erreichen. Dabei zeigt sich hier besonders stark ein bisher unerwähnter Aspekt des Schulversagens, nämlich die Frage nach der sozialen Gerechtigkeit: Hat doch, wie wir oben sahen, die Lesekompetenz einen Zusammenhang mit der sozialen Herkunft, gemessen am Ausbildungsniveau der El- 
tern, und weist das erreichte Schulniveau selbst, wie anderswo nachgewiesen (Notter, 1997), auch einen direkten Zusammenhang mit der sozialen Herkunft auf.

Die Ergebnisse dieser Analysen werfen unbequeme, grundsätzliche Fragen auf, die das Selbstverständnis der Institution Schule berühren. Der internationale Vergleich des Prozentsatzes der einheimischen Bevölkerung auf Lesekompetenzniveau 1 zeigt einerseits, dass das Schweizer Schulsystem im Vergleich zu anderen Ländern, wie z. B. Schweden, noch einen grossen Spielraum für mögliche Verbesserungen des Schulerfolgs hat. Die Tatsache andererseits, dass alle Länder, die grösstenteils eine lange Tradition der allgemeinen Volksschule haben, das Grundziel der Volksschule, die Alphabetisierung der gesamten Bevölkerung, noch nicht erreicht haben, wirft die Frage auf, wie weit dieses Grundziel überhaupt erreichbar ist. Der Optimismus, mit dem die offizielle Schweiz jahrelang gegenüber internationalen Organisationen wie z. B. der UNESCO die Alphabetisierungsrate der Bevölkerung mit 99.9 Prozent angab, dürfte auf jeden Fall fehl am Platz sein. Falls aber das Ziel der Alphabetisierung nicht erreichbar ist, stellt sich andererseits die Frage, mit welchem Recht gewisse Kinder und Jugendliche gezwungen werden, neun Jahre ihres Lebens einer Schule zu opfern, die ihnen nicht einmal die Garantie gibt, danach Lesen, Schreiben und Rechnen zu können, zumal ein Misserfolg zu einem grossen Teil durch ihre soziale Herkunft vorbestimmt ist.

\section{Literatur}

Drecoll, F. \& Müller, H. M. (Hrsg.). (1981). Für ein Recht auf Lesen. Frankfurt a. M.: Diesterweg.

Giere, U. (1987). Functional Illiteracy in Industrialized Countries: An Analytical Bibliography. Hamburg: UNESCO Institute for Education.

Hodel, I. (1985) Analphabetismus bei Jugendlichen und erwachsenen Schweizern: Erste Aussagen anhand von Umfragen in schweizerischen Institutionen. Unveröff. Lizentiatsarbeit, Universität Freiburg (Schweiz), Philosophische Fakultät.

Kirsch, I. S. \& Mosenthal, P. B. (1990). Exploring document literacy: Variables underlying the performance of young adults. Reading Research Quarterly, XXV, (1), 5-30.

Notter, Ph., Bonerad, E.-M. \& Stoll, F. (Hrsg.). (1999). Lesen - eine Selbstverständlichkeit? Schweizer Bericht zum "International Adult Literacy Survey». Chur: Rüegger.

Notter, Ph. (1996). Funktionaler Analphabetismus in der Schule? In Ph. Notter, U. Meier-Civelli, J. W. Nieuwenboom, P. Rüesch \& F. Stoll (1996). Lernziel Lesen. Lesekompetenzen von Kindern und Jugendlichen in der Schweiz. Schweizer Bericht über die IEA Reading Literacy Study. Aarau: Sauerländer.

Notter, Ph. (1997). Ausbildung und Lesekompetenzen. Eine Analyse aufgrund der Schweizer Daten des «International Adult Literacy Survey». Bildungsforschung und Bildungspraxis, 19, (3), 292-314.

OECD \& Statistics Canada (1995). Literacy, Economy and Society. Results of the first International Adult Literacy Survey. Paris: OECD. (frz.: Littératie, Economie et Société / Résultats de la première Enquête internationale sur l'alphabétisation des adultes. Paris: OCDE). 
OECD \& HRDC [Human Resources Development Canada] (1997). Literacy Skills for the Knowledge Society. Further Results from the International Adult Literacy Survey. Paris: OECD. (frz.: Littératie et société du savoir. Nouveaux résultats de l'Enquête internationale sur les capacités de lecture et d'écriture des adultes. Paris: OCDE).

OECD \& Statistics Canada (2000). Literacy in the Information Age. Final Report of the International Adult Literacy Survey. Paris: OECD. (frz.: La littératie à l'ère de l'information. Rapport final de l'enquête sur la littératie des adultes. Paris: OCDE).

Tuijnman, A. (2001). Benchmarking Adult Literacy in North America: An International Comparative Study. Ottawa: Statistics Canada.

\section{L'échec scolaire en tant qu'insuffisance de compétences en lecture et en calcul}

Résumé

Cette contribution envisage l'échec scolaire sous l'angle de la maîtrise insuffisante de compétences en lecture et en calcul après une scolarisation de plusieurs années. Elle analyse pour cela les résultats de l'enquête internationale «International Adult Literacy Survey » qui a étudié les compétences en lecture et en calcul d'une population d'adultes. Deux questions sont posées : 1. Combien de personnes en Suisse présentent des niveaux de compétence insuffisants en lecture et calcul ? 2. Doit-considérer que les personnes dont le niveau de formation ne dépasse pas l'école obligatoire forment un groupe à risque ?

Au sein de la population autochtone, environ $14 \%$ des adultes présentent dans au moins un des trois domaines considérés dans l'étude des niveaux de compétences insuffisants par rapport aux exigences de notre société. Cette proportion diminue à peine chez les jeunes; près de $6 \%$ de ceux-ci démontrent des compétences insuffisantes dans au moins un domaine. Parmi les personnes qui n'ont pas poursuivi leur formation au-delà de l'école obligatoire, cette proportion se monte à 39\%. Elles peuvent alors être considérées comme formant un groupe à risque.

\section{L'insuccesso scolastico come carenza di competenze in matematica e nella lettura}

\section{Riassunto}

Il contributo affronta la questione dell'insuccesso scolastico nella lettura e in matematica defininendolo in termini di carenza di competenze dopo una frequenza scolastica di più anni. Vengono presi in considerazione i dati della «International Adult Literacy Survey» che ha indagato queste competenze nella popolazione adulta. Due sono gli interrogativi posti. In primo luogo: quanto sono diffuse le carenze di competenza in matematica e nella lettura in Svizzera? In secondo 
luogo: occorre considerare coloro che hanno concluso la formazione alla fina del secondario I come un gruppo a rischio? Della popolazione indigena ca. il 14\% presenta carenze significative in almeno un dei tre ambiti di competenza indagati. La percentuale diminuisce con l'età, tuttavia anche nei giovani resta un $6 \%$ con le stesse difficoltà. Nel gruppo di persone che hanno concluso la loro carriera scolastica alla fine del secondario I ca. il 39\% manifesta carenze in almeno una delle competenze in questione. Di conseguenza questo gruppo deve essere considerato a rischio.

\section{School Failure as Insufficient Reading and Math Competence}

\section{Summary}

The findings of this study tie school failure to a lack of competence in reading and math, even after many years of schooling. The author analyzes the results of the "International Adult Literacy Survey" which investigated the reading and math competence of adults. Two questions are raised: 1 . How widespread is the problem of insufficient reading and math skills in Switzerland? and, 2. Are those pupils who do not go any further than secondary school level 1 (9th grade) at risk? Approximately $14 \%$ of the native population show insufficient skills for today's society in at least three of the examined fields. Though the figure is lower for younger people, approximately $6 \%$ of them still show a lack of competence in at least one of the areas. For the general segment of the population which completed no more than level 1 of secondary school, at least 39\% show a lack of skills in at least one area. This is the group that should be considered at risk. 